PROCEEDINGS OF THE

AMERICAN MATHEMATICAL SOCIETY

Volume 134, Number 9, September 2006, Pages 2621-2631

S 0002-9939(06)08251-7

Article electronically published on February 17, 2006

\title{
ARENS-MICHAEL ENVELOPING ALGEBRAS AND ANALYTIC SMASH PRODUCTS
}

\author{
A. YU. PIRKOVSKII
}

(Communicated by Martin Lorenz)

\begin{abstract}
Let $\mathfrak{g}$ be a finite-dimensional complex Lie algebra, and let $U(\mathfrak{g})$ be its universal enveloping algebra. We prove that if $\widehat{U}(\mathfrak{g})$, the Arens-Michael envelope of $U(\mathfrak{g})$ is stably flat over $U(\mathfrak{g})$ (i.e., if the canonical homomorphism $U(\mathfrak{g}) \rightarrow \widehat{U}(\mathfrak{g})$ is a localization in the sense of Taylor (1972), then $\mathfrak{g}$ is solvable. To this end, given a cocommutative Hopf algebra $H$ and an $H$-module algebra $A$, we explicitly describe the Arens-Michael envelope of the smash product $A \# H$ as an "analytic smash product" of their completions w.r.t. certain families of seminorms.
\end{abstract}

The Arens-Michael envelope of a complex associative algebra $A$ is defined as the completion of $A$ w.r.t. the family of all submultiplicative seminorms on $A$. This notion (under a different name) was introduced by Taylor [15, and the terminology "Arens-Michael envelope" is due to Helemskii [5]. An important example is the polynomial algebra $\mathbb{C}\left[t_{1}, \ldots, t_{n}\right]$ whose Arens-Michael envelope is isomorphic to the algebra $\mathcal{O}\left(\mathbb{C}^{n}\right)$ of entire functions endowed with the compact-open topology. Thus the Arens-Michael envelope of a noncommutative finitely generated algebra can be viewed as an "algebra of noncommutative entire functions" (cf. [16, 17]).

Given an algebra $A$, it is natural to ask to what extent homological properties of its Arens-Michael envelope $\widehat{A}$ (considered as a topological algebra) are related to those of $A$. To handle this problem, it is convenient to use the notion of localization. Roughly speaking, a topological algebra homomorphism $A \rightarrow B$ is a localization if it identifies the category of topological $B$-modules with a full subcategory of the category of topological $A$-modules, and if the homological relations between $B$-modules do not change when the modules are considered as $A$-modules. Localizations were introduced by Taylor [16] in connection with the functional calculus problem for several commuting Banach space operators. A purely algebraic counterpart of this notion was studied by Neeman and Ranicki 10. (Note that their terminology differs from Taylor's terminology; namely, a homomorphism $A \rightarrow B$ is a localization in Taylor's sense precisely when $B$ is stably flat over $A$ in the sense of Neeman and Ranicki.)

Thus a natural question is whether or not $\widehat{A}$ is stably flat over $A$. Taylor [16] proved that this is the case for $A=\mathbb{C}\left[t_{1}, \ldots, t_{n}\right]$ and for $A=F_{n}$, the free algebra on $n$ generators. In the case where $A=U(\mathfrak{g})$, the universal enveloping algebra of a

Received by the editors July 20, 2004 and, in revised form, March 24, 2005.

2000 Mathematics Subject Classification. Primary 46M18, 46H05, 16S30, 16S40, $18 \mathrm{G} 25$.

This work was partially supported by the RFBR grants 05-01-00982 and 05-01-00001, and by the President of Russia grant MK-2049.2004.1. 
complex Lie algebra $\mathfrak{g}$, Dosiev [3] proved that $\widehat{U}(\mathfrak{g})$ is stably flat over $U(\mathfrak{g})$ provided $\mathfrak{g}$ is metabelian. In [11] we extended this result to the case where $\mathfrak{g}$ admits a positive grading. On the other hand, Taylor [16] showed that $\widehat{U}(\mathfrak{g})$ is not stably flat over $U(\mathfrak{g})$ if $\mathfrak{g}$ is semisimple. Here we generalize this result and show that $\widehat{U}(\mathfrak{g})$ can be stably flat over $U(\mathfrak{g})$ only when $\mathfrak{g}$ is solvable.

Our approach is based on a notion of an "analytic smash product", which is a continuous version of the corresponding algebraic notion [14]. To prove the abovementioned result, we first show that for each cocommutative Hopf algebra $H$ and each $H$-module algebra $A$ the Arens-Michael envelope of the (algebraic) smash product $A \# H$ is isomorphic to the analytic smash product of $\widehat{H}$ and the completion of $A$ w.r.t. a certain family of seminorms determined by the action of $H$.

Remark. A. Dosiev has kindly informed the author that he proved the stable flatness of the Arens-Michael envelope $\widehat{U}(\mathfrak{g})$ over $U(\mathfrak{g})$ under the condition that $\mathfrak{g}$ is a nilpotent Lie algebra with normal growth. Roughly speaking, $\mathfrak{g}$ has normal growth if for each embedding of $\mathfrak{g}$ into a Banach algebra, norms of powers of elements from $[\mathfrak{g}, \mathfrak{g}]$ decrease sufficiently rapidly. The class of Lie algebras with normal growth contains all metabelian Lie algebras, but it is not clear how this class is related to that of positively graded Lie algebras.

\section{Preliminaries}

We shall work over the complex numbers $\mathbb{C}$. All associative algebras and algebra homomorphisms are assumed to be unital.

By a topological algebra we mean a topological vector space $A$ together with the structure of an associative algebra such that the multiplication map $A \times A \rightarrow A$ is separately continuous. A complete, Hausdorff, locally convex topological algebra with jointly continuous multiplication is called a $\widehat{\otimes}$-algebra (see [15, 4]). If $A$ is a $\widehat{\otimes}$-algebra, then the multiplication $A \times A \rightarrow A$ extends to a linear continuous map from the completed projective tensor product $A \widehat{\otimes} A$ to $A$. In other words, a $\widehat{\otimes}$-algebra is just an algebra in the tensor category $(\mathbf{L C S}, \widehat{\otimes})$ of complete Hausdorff locally convex spaces. The latter observation can be used to define $\widehat{\otimes}$-coalgebras, $\widehat{\otimes}$-bialgebras, and Hopf $\widehat{\otimes}$-algebras; see, e.g., 1 .

Recall that a seminorm $\|\cdot\|$ on an algebra $A$ is called submultiplicative if $\|a b\| \leq$ $\|a\|\|b\|$ for all $a, b \in A$. This means precisely that the corresponding unit ball $U=\{a \in A:\|a\| \leq 1\}$ is idempotent, i.e., satisfies $U^{2} \subset U$. A topological algebra $A$ is said to be locally $m$-convex if its topology can be defined by a family of submultiplicative seminorms. Note that the multiplication in a locally $m$-convex algebra is jointly continuous. An Arens-Michael algebra is a complete, Hausdorff, locally $m$-convex algebra.

The following useful lemma is due to Mitiagin, Rolewicz, and Żelazko 9 .

Lemma 1.1. Let $A$ be a locally convex algebra with topology generated by a family $\left\{\|\cdot\|_{\nu}: \nu \in \Lambda\right\}$ of seminorms. Suppose that for each $\nu \in \Lambda$ there exist $\mu \in \Lambda$ and $C>0$ such that $\left\|a_{1} \cdots a_{n}\right\|_{\nu} \leq C^{n}\left\|a_{1}\right\|_{\mu} \cdots\left\|a_{n}\right\|_{\mu}$ for each $a_{1}, \ldots, a_{n} \in A$. Then $A$ is locally $m$-convex.

Corollary 1.2. Let $A$ be a locally convex algebra with topology generated by a family $\left\{\|\cdot\|_{\nu}: \nu \in \Lambda\right\}$ of seminorms. Suppose that for each $\nu \in \Lambda$ there exist $\mu \in \Lambda$ and $C>0$ such that $\|a b\|_{\nu} \leq C\|a\|_{\mu}\|b\|_{\nu}$ for each $a, b \in A$. Then $A$ is locally $m$-convex. 
We shall use the latter corollary in the following geometric form.

Corollary 1.3. Let $A$ be a locally convex algebra. Suppose that $A$ has a base $\mathcal{U}$ of absolutely convex 0-neighborhoods with the property that for each $V \in \mathcal{U}$ there exist $U \in \mathcal{U}$ and $C>0$ such that $U V \subset C V$. Then $A$ is locally m-convex.

Let $A$ be a topological algebra. A pair $\left(\widehat{A}, \iota_{A}\right)$ consisting of an Arens-Michael algebra $\widehat{A}$ and a continuous homomorphism $\iota_{A}: A \rightarrow \widehat{A}$ is called the Arens-Michael envelope of $A$ [15, 5] if for each Arens-Michael algebra $B$ and for each continuous homomorphism $\varphi: A \rightarrow B$ there exists a unique continuous homomorphism $\widehat{\varphi}: \widehat{A} \rightarrow$ $B$ making the following diagram commutative:

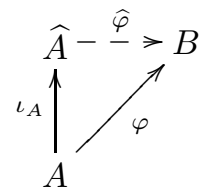

In the above situation, we say that $\widehat{\varphi}$ extends $\varphi$ (though $\iota_{A}$ is not injective in general; see [5] or [1] for details).

Recall (see [15] and [5, Chap. V]) that the Arens-Michael envelope of a topological algebra $A$ always exists and can be obtained as the completion 11 of $A$ w.r.t. the family of all continuous submultiplicative seminorms on $A$. This implies, in particular, that $\iota_{A}: A \rightarrow \widehat{A}$ has dense range. Clearly, the Arens-Michael envelope is unique in the obvious sense.

Each associative algebra $A$ becomes a topological algebra w.r.t. the finest locally convex topology. The Arens-Michael envelope, $\widehat{A}$, of the resulting topological algebra will be referred to as the Arens-Michael envelope of $A$. That is, $\widehat{A}$ is the completion of $A$ w.r.t. the family of all submultiplicative seminorms. Thus a neighborhood base at 0 for the topology on $A$ inherited from $\widehat{A}$ consists of all absorbing, idempotent, absolutely convex subsets.

Here is a basic example: the Arens-Michael envelope of the polynomial algebra $\mathbb{C}\left[t_{1}, \ldots, t_{n}\right]$ is topologically isomorphic to the algebra $\mathcal{O}\left(\mathbb{C}^{n}\right)$ of entire functions endowed with the compact-open topology [15]. For other examples, see [15, 16, 5, 11.

Let $\mathfrak{g}$ be a finite-dimensional complex Lie algebra. We may define the ArensMichael enveloping algebra of $\mathfrak{g}$ as a pair $\left(\widehat{U}(\mathfrak{g}), \iota_{\mathfrak{g}}\right)$ consisting of an Arens-Michael algebra $\widehat{U}(\mathfrak{g})$ and a Lie algebra homomorphism $\iota_{\mathfrak{g}}: \mathfrak{g} \rightarrow \widehat{U}(\mathfrak{g})$ such that for each Arens-Michael algebra $B$ and for each Lie algebra homomorphism $\varphi: \mathfrak{g} \rightarrow B$ there exists a unique $\widehat{\otimes}$-algebra homomorphism $\widehat{\varphi}: \widehat{U}(\mathfrak{g}) \rightarrow B$ such that $\widehat{\varphi} \iota_{\mathfrak{g}}=\varphi$. Clearly, $\widehat{U}(\mathfrak{g})$ is nothing but the Arens-Michael envelope of $U(\mathfrak{g})$, the universal enveloping algebra of $\mathfrak{g}$.

If $H$ is a bialgebra (resp., a Hopf algebra), then it is easy to show that the Arens-Michael envelope $\widehat{H}$ is a $\widehat{\otimes}$-bialgebra (resp., a Hopf $\widehat{\otimes}$-algebra) in a natural way (for details, see [1]).

Let $A$ be a $\widehat{\otimes}$-algebra. A left $A$ - $\widehat{\otimes}$-module is a complete Hausdorff locally convex space $X$ together with the structure of a left unital $A$-module such that the map $A \times X \rightarrow X,(a, x) \mapsto a \cdot x$ is jointly continuous. As above, this means precisely

\footnotetext{
${ }^{1}$ Here we follow the convention that the completion of a non-Hausdorff locally convex space $E$ is defined to be the completion of the corresponding Hausdorff space $E / \overline{\{0\}}$.
} 
that $X$ is a left $A$-module in $(\mathbf{L C S}, \widehat{\otimes})$. If $X$ and $Y$ are left $A$ - $\widehat{\otimes}$-modules, then the vector space of all (continuous) $A$-module morphisms from $X$ to $Y$ is denoted by ${ }_{A} \mathbf{h}(X, Y)$. Left $A$ - $\widehat{\otimes}$-modules and their (continuous) morphisms form a category denoted by $A$-mod. Right $A$ - $\widehat{\otimes}$-modules, $A$ - $\widehat{\otimes}$-bimodules, and their morphisms are defined similarly. The corresponding categories are denoted by $\bmod -A$ and $A$-mod- $A$, respectively.

If $X$ is a right $A-\widehat{\otimes}$-module and $Y$ is a left $A$ - $\widehat{\otimes}$-module, then their $A$-module tensor product $X \widehat{\otimes}_{A} Y$ is defined to be the completion of the quotient $(X \widehat{\otimes} Y) / N$, where $N \subset X \widehat{\otimes} Y$ is the closed linear span of all elements of the form $x \cdot a \otimes y-x \otimes a \cdot y$ $(x \in X, y \in Y, a \in A) \sqrt{2}$. As in pure algebra, the $A$-module tensor product can be characterized by a certain universal property (see 4 for details).

A morphism $\sigma: X \rightarrow Y$ of left $A$ - $\widehat{\otimes}$-modules is said to be an admissible epimorphism if there exists a linear continuous map $\tau: Y \rightarrow X$ such that $\sigma \tau=\mathbf{1}_{Y}$, i.e., if $\sigma$ is a retraction when considered in the category of topological vector spaces. A chain complex $X_{\bullet}=\left(X_{n}, d_{n}\right)$ of left $A$ - $\widehat{\otimes}$-modules is called admissible if it splits as a complex of topological vector spaces. Equivalently, $X_{\bullet}$ is admissible if each $d_{n}$ is an admissible epimorphism of $X_{n+1}$ onto $\operatorname{Ker} d_{n-1} \subset X_{n}$.

An $A$-module $P \in A$-mod is called projective if for each admissible epimorphism $X \rightarrow Y$ in $A$-mod the induced map ${ }_{A} \mathbf{h}(P, X) \rightarrow{ }_{A} \mathbf{h}(P, Y)$ is surjective. Given a left $A$-ब्ब-module $X$, a projective resolution of $X$ is a chain complex $P_{\bullet}=\left(P_{n}, d_{n}\right)_{n \geq 0}$ consisting of projective left $A$ - $\widehat{\otimes}$-modules $P_{n}$ together with a morphism $\epsilon: P_{0} \rightarrow X$ such that the augmented sequence

$$
0 \leftarrow X \stackrel{\epsilon}{\leftarrow} P_{0} \stackrel{d_{0}}{\longleftarrow} \cdots \leftarrow P_{n} \stackrel{d_{n}}{\longleftarrow} P_{n+1} \leftarrow \cdots
$$

is an admissible complex. The category $A$-mod has enough projectives, i.e., each $A$ - $\widehat{\otimes}$-module has a projective resolution [4. Therefore one can define the derived functors Ext and Tor following the general patterns of relative homological algebra. For details, see [4].

Similar definitions apply to right $A-\widehat{\otimes}$-modules and to $A$ - $\widehat{\otimes}$-bimodules. A projective resolution of $A$ considered as a $\widehat{\otimes}$-bimodule over itself is called a projective bimodule resolution of $A$.

Let $A$ and $B$ be $\widehat{\otimes}$-algebras and $\theta: A \rightarrow B$ a continuous homomorphism. Following Taylor [16], we say that $\theta$ is a localization if the following conditions are satisfied:

(i) There exists a projective bimodule resolution $P_{\bullet} \rightarrow A \rightarrow 0$ of $A$ such that the complex

$$
B \underset{A}{\widehat{\otimes}} P \bullet \widehat{\otimes}_{A} B \rightarrow \underset{A}{\widehat{\otimes}} A \underset{A}{\widehat{\otimes}} B \cong B \underset{A}{\widehat{\otimes}} B \rightarrow 0
$$

is admissible.

(ii) The map $B \widehat{\otimes}_{A} B \rightarrow B, b_{1} \otimes b_{2} \mapsto b_{1} b_{2}$ is a topological isomorphism.

In this situation, we say (following Neeman and Ranicki [10]) that $B$ is stably flat over $A$.

The following observation is due to Taylor [16].

\footnotetext{
${ }^{2}$ To avoid confusion, we note that this definition of $X \widehat{\otimes}_{A} Y$ (due to Rieffel 12 and Helemskii (4) ) is different from that given by Kiehl and Verdier [6] and Taylor [15. More precisely, $X \widehat{\otimes}_{A} Y$ is the completion of the Kiehl-Verdier-Taylor tensor product.
} 
Proposition 1.4. Let $A \rightarrow B$ be a localization. Then for each $M \in \bmod -B$ and each $N \in B$-mod there are natural isomorphisms

$$
\operatorname{Tor}_{n}^{A}(M, N) \cong \operatorname{Tor}_{n}^{B}(M, N) \quad(n \geq 0) .
$$

For later reference, let us recall a standard notation from the theory of topological vector spaces. Let $E$ and $F$ be locally convex spaces. For each 0-neighborhood $U \subset E$ and each 0-neighborhood $V \subset F$ let $\Gamma(U \otimes V)$ denote the absolutely convex hull of the set

$$
U \otimes V=\{u \otimes v: u \in U, v \in V\} \subset E \otimes F .
$$

Then all sets of the form $\Gamma(U \otimes V)$ form a base of 0-neighborhoods for the projective tensor product topology on $E \otimes F$.

\section{Algebraic and analytic Smash products}

Let $H$ be a bialgebra. Recall that an $H$-module algebra is an algebra $A$ endowed with the structure of a left $H$-module such that the product $A \otimes A \rightarrow A$ and the unit map $\mathbb{C} \rightarrow A$ are $H$-module morphisms. For example, if $\mathfrak{g}$ is a Lie algebra acting on $A$ by derivations, then the action $\mathfrak{g} \times A \rightarrow A$ extends to a map $U(\mathfrak{g}) \times A \rightarrow A$ making $A$ into a $U(\mathfrak{g})$-module algebra. Similarly, if $G$ is a group acting on $A$ by automorphisms, then $A$ becomes a $\mathbb{C} G$-module algebra, where $\mathbb{C} G$ denotes the group algebra of $G$.

Given an $H$-module algebra $A$, the smash product algebra $A \# H$ is defined as follows (see, e.g., [14). As a vector space, $A \# H$ is equal to $A \otimes H$. To define multiplication, denote by $\mu_{H, A}: H \otimes A \rightarrow A$ the action of $H$ on $A$, and define $\tau: H \otimes A \rightarrow A \otimes H$ as the composition

$$
H \otimes A \stackrel{\Delta_{H} \otimes 1_{A}}{\longrightarrow} H \otimes H \otimes A \stackrel{1_{H} \otimes c_{H, A}}{\longrightarrow} H \otimes A \otimes H \stackrel{\mu_{H, A} \otimes 1_{H}}{\longrightarrow} A \otimes H
$$

(here $c_{H, A}$ denotes the flip $H \otimes A \rightarrow A \otimes H$ ). Then the map

$$
(A \otimes H) \otimes(A \otimes H) \stackrel{1_{A} \otimes \tau \otimes 1_{H}}{\longrightarrow} A \otimes A \otimes H \otimes H \stackrel{\mu_{A} \otimes \mu_{H}}{\longrightarrow} A \otimes H
$$

is an associative multiplication on $A \otimes H$. The resulting algebra is denoted by $A \# H$ and is called the smash product of $A$ with $H$. For later reference, note that the maps $i_{1}: A \rightarrow A \# H, i_{1}(a)=a \otimes 1$ and $i_{2}: H \rightarrow A \# H, i_{2}(h)=1 \otimes h$ are algebra homomorphisms.

Similar definitions apply in the $\widehat{\otimes}$-algebra case. Namely, if $H$ is a $\widehat{\otimes}$-bialgebra, then an $H$ - $\widehat{\otimes}$-module algebra is a $\widehat{\otimes}$-algebra $A$ together with the structure of a left $H$-ब्ब-module such that the product $A \widehat{\otimes} A \rightarrow A$ and the unit map $\mathbb{C} \rightarrow A$ are $H$ module morphisms. We define the analytic smash product $A \widehat{\#} H$ to be $A \widehat{\otimes} H$ as a locally convex space. By replacing $\otimes$ by $\widehat{\otimes}$ in (1) and (2), we obtain a multiplication on $A \widehat{\#} H$ making it into a $\widehat{\otimes}$-algebra.

Example 2.1. Let $A$ be a Banach algebra, and let $G$ be a discrete group acting on $A$ by isometric automorphisms. Then $A \widehat{\#} \ell^{1}(G)$ is isomorphic to the covariance algebra $\ell_{A}^{1}(G)$ introduced by Doplicher, Kastler, and Robinson [2] in a more general setting of locally compact groups.

For numerous related constructions and references, see [13].

Let $E$ be a vector space, and let $\mathcal{T}$ be a set of linear operators on $E$. 
Definition 2.1. We say that a seminorm $\|\cdot\|$ on $E$ is $\mathcal{T}$-stable if for each $T \in \mathcal{T}$ there exists $C>0$ such that $\|T x\| \leq C\|x\|$ for each $x \in E$. A subset $U \subset E$ is said to be $\mathcal{T}$-stable if for each $T \in \mathcal{T}$ there exists $C>0$ such that $T(U) \subset C U$.

Clearly, a seminorm $\|\cdot\|$ is $\mathcal{T}$-stable if and only if the unit ball $\{x \in E:\|x\| \leq 1\}$ is $\mathcal{T}$-stable.

If $E$ is a left module over an associative algebra $B$, then we say that a seminorm $\|\cdot\|$ on $E$ (resp. a subset $U \subset E$ ) is $B$-stable if it is stable w.r.t. the set of operators $\{x \mapsto b \cdot x: b \in B\}$. Similar definitions apply in the case where $E$ is a left module over a Lie algebra $\mathfrak{g}$ or a left module over a group $G$. Note that if a subset $M \subset B$ generates $B$ as an algebra, then a seminorm $\|\cdot\|$ on $E$ is $B$-stable if and only if it is $M$-stable. In particular, a seminorm on a $\mathfrak{g}$-module (resp., on a $G$-module) is $U(\mathfrak{g})$-stable (resp., $\mathbb{C} G$-stable) if and only if it is $\mathfrak{g}$-stable (resp., $G$-stable).

Definition 2.2. Let $H$ be a bialgebra and $A$ an $H$-module algebra. We define the $H$-completion $\widetilde{A}$ to be the completion of $A$ w.r.t. the family of all $H$-stable, submultiplicative seminorms.

It is immediate from the definition that $\widetilde{A}$ is an Arens-Michael algebra.

Proposition 2.1. Let $H$ be a bialgebra and $A$ an $H$-module algebra. Then the action of $H$ on $A$ uniquely extends to an action of $\widehat{H}$ on $\widetilde{A}$, so that $\widetilde{A}$ becomes an $\widehat{H}-\widehat{\otimes}$-module algebra. Moreover, the smash product $\widetilde{A} \widehat{\#} \widehat{H}$ is an Arens-Michael algebra.

Proof. Let us endow $H$ and $A$ with the topologies inherited from $\widehat{H}$ and $\widetilde{A}$, respectively. In order to prove the first assertion, it suffices to show that the action $H \times A \rightarrow A$ is jointly continuous. Let $\|\cdot\|$ be an $H$-stable, submultiplicative seminorm on $A$. For each $h \in H$, set $\|h\|^{\prime}=\sup \{\|h \cdot a\|:\|a\| \leq 1\}$. Since $\|\cdot\|$ is $H$-stable, it follows that $\|\cdot\|^{\prime}$ is a well-defined seminorm on $H$. We obviously have $\left\|h_{1} h_{2}\right\|^{\prime} \leq\left\|h_{1}\right\|^{\prime}\left\|h_{2}\right\|^{\prime}$ for all $h_{1}, h_{2} \in H$, and $\|h \cdot a\| \leq\|h\|^{\prime}\|a\|$ for each $h \in H, a \in A$. Hence the action $H \times A \rightarrow A$ is jointly continuous, so it uniquely extends to a jointly continuous bilinear map $\widehat{H} \times \widetilde{A} \rightarrow \widetilde{A}$. Since the canonical image of $H$ (resp., $A$ ) is dense in $\widehat{H}$ (resp., in $\widetilde{A}$ ), it follows that $\widetilde{A}$ becomes an $\widehat{H}-\widehat{\otimes}$-module algebra.

To prove that $\widetilde{A} \widehat{\#} \widehat{H}$ is an Arens-Michael algebra, it suffices to show that the algebraic smash product $A \# H$ is locally $m$-convex w.r.t. the projective tensor product topology. Recall that a typical 0-neighborhood in $A \# H$ has the form $\Gamma(U \otimes V)$, where $U \subset A$ and $V \subset H$ are absorbing, absolutely convex, idempotent subsets, and $U$ is $H$-stable. Given such $U$ and $V$, define

$$
W=\{w \in H: w \cdot U \subset U\}
$$

It is easy to see that $W$ is absorbing, absolutely convex, and idempotent, so it is a 0-neighborhood in $H$. Set $V^{\prime}=\Delta^{-1}(\Gamma(W \otimes V))$, where $\Delta: H \rightarrow H \otimes H$ is the comultiplication on $H$. We claim that

$$
\Gamma\left(U \otimes V^{\prime}\right) \Gamma(U \otimes V) \subset \Gamma(U \otimes V) .
$$


Indeed, take $u_{1}, u_{2} \in U, v_{1} \in V^{\prime}$, and $v_{2} \in V$. Then $\Delta\left(v_{1}\right) \in \Gamma(W \otimes V)$. Since $W \cdot U \subset U$, i.e., $\mu_{H, A}(\Gamma(W \otimes U)) \subset U$, it follows that

$$
\begin{aligned}
& \tau\left(v_{1} \otimes u_{2}\right) \in\left(\mu_{H, A} \otimes 1_{H}\right)\left(1_{H} \otimes c_{H, A}\right)(\Gamma(W \otimes V \otimes U)) \\
&=\left(\mu_{H, A} \otimes 1_{H}\right)(\Gamma(W \otimes U \otimes V)) \subset \Gamma(U \otimes V) .
\end{aligned}
$$

Therefore,

$$
\begin{aligned}
\left(u_{1} \otimes v_{1}\right)\left(u_{2} \otimes v_{2}\right) & =\left(\mu_{A} \otimes \mu_{H}\right)\left(u_{1} \otimes \tau\left(v_{1} \otimes u_{2}\right) \otimes v_{2}\right) \\
& \in\left(\mu_{A} \otimes \mu_{H}\right)(U \otimes \Gamma(U \otimes V) \otimes V) \subset \Gamma(U \otimes V) .
\end{aligned}
$$

This proves (3). Together with Corollary 1.3, this implies that $A \# H$ is locally $m$-convex, so that $\widetilde{A} \widehat{\#} \widehat{H}$ is an Arens-Michael algebra.

Theorem 2.2. Let $H$ be a cocommutative Hopf algebra and $A$ an $H$-module algebra. Then the canonical map $A \# H \rightarrow \widetilde{A} \widehat{\#} \widehat{H}$ extends to a $\widehat{\otimes}$-algebra isomorphism

$$
(A \# H)^{\wedge} \cong \widetilde{A} \widehat{\#} \widehat{H} \text {. }
$$

Proof. Let $\varphi: A \# H \rightarrow B$ be a homomorphism to an Arens-Michael algebra $B$. As before, we endow $A$ and $H$ with the topologies inherited from $\widetilde{A}$ and $\widehat{H}$, respectively. Since the canonical image of $A \# H$ is dense in $\widetilde{A} \widehat{\#} \widehat{H}$, it suffices to show that $\varphi$ is continuous w.r.t. the projective tensor product topology on $A \# H$.

Define homomorphisms $\varphi_{1}: A \rightarrow B$ and $\varphi_{2}: H \rightarrow B$ by $\varphi_{1}(a)=\varphi(a \otimes 1)$ and $\varphi_{2}(h)=\varphi(1 \otimes h)$. Then

$$
\varphi(a \otimes h)=\varphi((a \otimes 1)(1 \otimes h))=\varphi_{1}(a) \varphi_{2}(h)
$$

for each $a \in A, h \in H$. Therefore we need only prove that $\varphi_{1}$ and $\varphi_{2}$ are continuous.

Let $\|\cdot\|$ be a continuous submultiplicative seminorm on $B$. Then the seminorms $a \mapsto\|a\|^{\prime}=\left\|\varphi_{1}(a)\right\|(a \in A)$ and $h \mapsto\|h\|^{\prime \prime}=\left\|\varphi_{2}(h)\right\|(h \in H)$ are submultiplicative. This implies, in particular, that $\varphi_{2}$ is continuous. To prove the continuity of $\varphi_{1}$, we have to show that $\|\cdot\|^{\prime}$ is $H$-stable.

Let $h \in H$ be a primitive element. Then for each $a \in A$ we have

$$
\begin{array}{r}
(1 \otimes h)(a \otimes 1)=\tau(h \otimes a)=\left(\mu_{H, A} \otimes 1_{H}\right)\left(1_{H} \otimes c_{H, A}\right)((h \otimes 1+1 \otimes h) \otimes a) \\
=\left(\mu_{H, A} \otimes 1_{H}\right)(h \otimes a \otimes 1+1 \otimes a \otimes h)=h \cdot a \otimes 1+a \otimes h .
\end{array}
$$

Therefore,

$$
\begin{aligned}
\|h \cdot a\|^{\prime} & =\|\varphi(h \cdot a \otimes 1)\| \\
& =\|\varphi((1 \otimes h)(a \otimes 1)-a \otimes h)\| \\
& \leq\|\varphi(1 \otimes h)\|\|\varphi(a \otimes 1)\|+\|\varphi(a \otimes h)\| \\
& =\left\|\varphi_{2}(h)\right\|\left\|\varphi_{1}(a)\right\|+\left\|\varphi_{1}(a) \varphi_{2}(h)\right\| \leq 2 C\left\|\varphi_{1}(a)\right\|=2 C\|a\|^{\prime},
\end{aligned}
$$

where $C=\left\|\varphi_{2}(h)\right\|$.

Now let $g \in H$ be a group-like element. Then for each $a \in A$ we have

$$
\begin{aligned}
(1 \otimes g)(a \otimes 1)=\tau(g \otimes a) & =\left(\mu_{H, A} \otimes 1_{H}\right)\left(1_{H} \otimes c_{H, A}\right)(g \otimes g \otimes a) \\
& =\left(\mu_{H, A} \otimes 1_{H}\right)(g \otimes a \otimes g)=g \cdot a \otimes g .
\end{aligned}
$$


Therefore,

$$
\begin{aligned}
\|g \cdot a\|^{\prime} & =\|\varphi(g \cdot a \otimes 1)\|=\left\|\varphi\left((1 \otimes g)(a \otimes 1)\left(1 \otimes g^{-1}\right)\right)\right\| \\
& \leq\|\varphi(1 \otimes g)\|\|\varphi(a \otimes 1)\|\left\|\varphi\left(1 \otimes g^{-1}\right)\right\| \\
& =\left\|\varphi_{2}(g)\right\|\left\|\varphi_{1}(a)\right\|\left\|\varphi_{2}\left(g^{-1}\right)\right\|=C\|a\|^{\prime},
\end{aligned}
$$

where $C=\left\|\varphi_{2}(g)\right\|\left\|\varphi_{2}\left(g^{-1}\right)\right\|$.

Since $H$ is cocommutative, it is generated by primitive and group-like elements [14, 13.1]. Therefore it follows from (4) and (5) that $\|\cdot\|^{\prime}$ is $H$-stable. Hence $\varphi_{1}$ is continuous. In view of the above remarks, $\varphi$ is also continuous, and so it uniquely extends to a $\widehat{\otimes}$-algebra homomorphism $\widetilde{A} \widehat{\#} \widehat{H} \rightarrow B$. This completes the proof.

Corollary 2.3. Let $\mathfrak{g}$ be a Lie algebra acting on an algebra $A$ by derivations. Then $(A \# U(\mathfrak{g}))^{\wedge} \cong \widetilde{A} \widehat{\#} \widehat{U}(\mathfrak{g})$ as $\widehat{\otimes}$-algebras.

Corollary 2.4. Let $G$ be a group acting on an algebra $A$ by automorphisms. Then $(A \# \mathbb{C} G)^{\wedge} \cong \widetilde{A} \widehat{\#} \widehat{\mathbb{C} G}$ as $\widehat{\otimes}$-algebras.

\section{THE MAIN RESUlT}

Let $H$ be a $\widehat{\otimes}$-bialgebra with counit $\varepsilon: H \rightarrow \mathbb{C}$, and let $A$ be an $H$ - $\widehat{\otimes}$-module algebra.

Lemma 3.1. Define $\tau: H \otimes A \rightarrow A \otimes H$ by (11). Then $\left(1_{A} \otimes \varepsilon\right) \tau=\mu_{H, A}$.

Proof. This is a direct computation:

$$
\begin{aligned}
\left(1_{A} \otimes \varepsilon\right) \tau=\left(1_{A} \otimes \varepsilon\right)\left(\mu_{H, A} \otimes 1_{H}\right)\left(1_{H} \otimes c_{H, A}\right)\left(\Delta \otimes 1_{A}\right) & \\
=\mu_{H, A}\left(1_{H} \otimes 1_{A} \otimes \varepsilon\right) & \left(1_{H} \otimes c_{H, A}\right)\left(\Delta \otimes 1_{A}\right) \\
& =\mu_{H, A}\left(1_{H} \otimes \varepsilon \otimes 1_{A}\right)\left(\Delta \otimes 1_{A}\right)=\mu_{H, A} .
\end{aligned}
$$

Lemma 3.2. There is a unique left $A \widehat{\#} H-\widehat{\otimes}$-module structure on $A$ such that

$$
(a \otimes 1) \cdot b=a b, \quad(1 \otimes h) \cdot b=h \cdot b
$$

for each $a, b \in A, h \in H$.

Proof. Consider the map $1_{A} \otimes \varepsilon: A \widehat{\#} H \rightarrow A$. Let us prove that $\operatorname{Ker}\left(1_{A} \otimes \varepsilon\right)$ is a left ideal of $A \widehat{\#} H$. In view of the direct sum decomposition $A \widehat{\otimes} H=(A \widehat{\otimes} \operatorname{Ker} \varepsilon) \oplus$ $(A \widehat{\otimes} \mathbb{C} 1)$, is suffices to show that $\left(a_{1} \otimes h_{1}\right)\left(a_{2} \otimes h_{2}\right) \in \operatorname{Ker}\left(1_{A} \otimes \varepsilon\right)$ whenever $h_{2} \in \operatorname{Ker} \varepsilon$. We have

$$
\begin{aligned}
& \left(a_{1} \otimes h_{1}\right)\left(a_{2} \otimes h_{2}\right)=\left(\mu_{A} \otimes \mu_{H}\right)\left(a_{1} \otimes \tau\left(h_{1} \otimes a_{2}\right) \otimes h_{2}\right) \\
& \quad \in\left(\mu_{A} \otimes \mu_{H}\right)\left(A \widehat{\otimes} A \widehat{\otimes} H \widehat{\otimes} \mathbb{C} h_{2}\right) \subset \operatorname{Ker}\left(1_{A} \otimes \varepsilon\right) .
\end{aligned}
$$

Therefore $\operatorname{Ker}\left(1_{A} \otimes \varepsilon\right)$ is a left ideal of $A \widehat{\#} H$, so that we can make $A$ into a left $A \widehat{\#} H$ - $\widehat{\otimes}$-module via the identification $A=(A \widehat{\#} H) / \operatorname{Ker}\left(1_{A} \otimes \varepsilon\right)$.

Now take $a, b \in A$ and $h \in H$. We have

$$
(a \otimes 1) \cdot b=\left(1_{A} \otimes \varepsilon\right)((a \otimes 1)(b \otimes 1))=\left(1_{A} \otimes \varepsilon\right)(a b \otimes 1)=a b .
$$


On the other hand, Lemma 3.1 implies that

$$
(1 \otimes h) \cdot b=\left(1_{A} \otimes \varepsilon\right)((1 \otimes h)(b \otimes 1))=\left(1_{A} \otimes \varepsilon\right)(\tau(h \otimes b))=h \cdot b .
$$

Hence conditions (6) are satisfied.

The uniqueness readily follows from the identity $a \otimes h=(a \otimes 1)(1 \otimes h)$.

From now on, we endow $A$ with the left $A \widehat{\#} H$ - $\widehat{\otimes}$-module structure defined in the previous lemma.

Lemma 3.3. Suppose that $\mathbb{C}$ is a projective left $H-\widehat{\otimes}$-module. Then $A$ is a projective left $A \widehat{\#} H$-module.

Proof. Since $\mathbb{C}$ is projective, there exists an $H$-module morphism $\lambda: \mathbb{C} \rightarrow H$ such that $\varepsilon \lambda=1_{\mathbb{C}}$. Then the element $x_{0}=\lambda(1)$ satisfies $\varepsilon\left(x_{0}\right)=1$ and $h x_{0}=\varepsilon(h) x_{0}$ for each $h \in H$. Consider the map

$$
\rho: A \rightarrow A \widehat{\# H}, \quad \rho(a)=a \otimes x_{0} .
$$

We claim that $\rho$ is a left $A \widehat{\# H} H$-module morphism. To prove the claim, it is convenient to consider $A \widehat{\#} H$ as a left $A$ - $\widehat{\otimes}$-module and as a left $H$ - $\widehat{\otimes}$-module via the embeddings $i_{1}: A \rightarrow A \widehat{\#} H$ and $i_{2}: H \rightarrow A \widehat{\#} H$ given by $a \mapsto a \otimes 1$ and $h \mapsto 1 \otimes h$, respectively. Thus we have to show that $\rho$ is an $A$-module morphism and an $H$-module morphism.

For each $a, b \in A$ we have

$$
\rho(a b)=a b \otimes x_{0}=(a \otimes 1)\left(b \otimes x_{0}\right)=a \cdot \rho(b),
$$

so that $\rho$ is an $A$-module morphism. Further, the relation $h x_{0}=\varepsilon(h) x_{0}$ implies that $\left(1_{A} \otimes \mu_{H}\right)\left(u \otimes x_{0}\right)=\left(1_{A} \otimes \varepsilon\right)(u) \otimes x_{0}$ for each $u \in A \widehat{\otimes} H$. Together with Lemma 3.1 this gives

$$
\begin{aligned}
h \cdot \rho(a)=(1 \otimes h)\left(a \otimes x_{0}\right)= & \left(1_{A} \otimes \mu_{H}\right)\left(\tau(h \otimes a) \otimes x_{0}\right) \\
& =\left(1_{A} \otimes \varepsilon\right)(\tau(h \otimes a)) \otimes x_{0}=h \cdot a \otimes x_{0}=\rho(h \cdot a)
\end{aligned}
$$

for each $a \in A, h \in H$. Therefore $\rho$ is an $H$-module morphism and hence an $A \widehat{\#} H$-module morphism. Finally, since $\varepsilon\left(x_{0}\right)=1$, we see that $\left(1_{A} \otimes \varepsilon\right) \rho=1_{A}$. Thus $A$ is a retract of $A \widehat{\#} H$ in $A \widehat{\#} H$-mod, so it is projective.

Now let $\mathfrak{g}$ be a finite-dimensional complex Lie algebra. Denote by $\mathfrak{r}$ the radical of $\mathfrak{g}$, and consider the Levi decomposition $\mathfrak{g}=\mathfrak{r} \oplus \mathfrak{h}$. The action of $\mathfrak{h}$ on $\mathfrak{r}$ by commutators extends to an action of $\mathfrak{h}$ on $U(\mathfrak{r})$ by derivations, and there exists a canonical isomorphism $U(\mathfrak{g}) \cong U(\mathfrak{r}) \# U(\mathfrak{h})$ (see, e.g., [8, 1.7.11]). Using Corollary 2.3, we see that

$$
\widehat{U}(\mathfrak{g}) \cong(U(\mathfrak{r}) \# U(\mathfrak{h})) \cong \widetilde{U}(\mathfrak{r}) \widehat{\#} \widehat{U}(\mathfrak{h}) .
$$

Lemma 3.4. $\widetilde{U}(\mathfrak{r})$ is a projective $\widehat{U}(\mathfrak{g})-\widehat{\otimes}$-module. As a corollary,

$$
\operatorname{Tor}_{k}^{\widehat{U}(\mathfrak{g})}(\mathbb{C}, \widetilde{U}(\mathfrak{r}))=0 \text { for each } k>0 .
$$

Proof. Since $\mathfrak{h}$ is semisimple, the Arens-Michael envelope $\widehat{U}(\mathfrak{h})$ is isomorphic to a direct product of full matrix algebras [16, Corollary 7.6]. Hence each $\widehat{U}(\mathfrak{h})-\widehat{\otimes}-$ module is projective [15] (see also [4, 5.28]). Now it remains to apply Lemma 3.3.

Lemma 3.5. Suppose that $k=\operatorname{dim} \mathfrak{h}>0$. Then $\operatorname{Tor}_{k}^{U(\mathfrak{g})}(\mathbb{C}, \widetilde{U}(\mathfrak{r})) \neq 0$. 
Proof. Set $A=\widetilde{U}(\mathfrak{r})$, and recall that the groups $H_{p}(\mathfrak{g}, A)=\operatorname{Tor}_{p}^{U(\mathfrak{g})}(\mathbb{C}, A)$ can be computed as the homology groups of the standard complex $C .(\mathfrak{g}, A)$ :

$$
0 \leftarrow A \stackrel{d}{\leftarrow} \mathfrak{g} \otimes A \stackrel{d}{\leftarrow} \bigwedge^{2} \mathfrak{g} \otimes A \stackrel{d}{\leftarrow} \cdots \bigwedge^{p-1} \mathfrak{g} \otimes A \stackrel{d}{\leftarrow} \bigwedge^{p} \mathfrak{g} \otimes A \stackrel{d}{\leftarrow} \cdots .
$$

The differential $d$ is given by

$$
\begin{aligned}
d\left(X_{1} \wedge\right. & \left.\cdots \wedge X_{p} \otimes a\right)=\sum_{i=1}^{p}(-1)^{i-1} X_{1} \wedge \cdots \wedge \hat{X}_{i} \wedge \cdots \wedge X_{p} \otimes X_{i} \cdot a \\
& +\sum_{1 \leq i<j \leq p}(-1)^{i+j}\left[X_{i}, X_{j}\right] \wedge X_{1} \wedge \cdots \wedge \hat{X}_{i} \wedge \cdots \wedge \hat{X}_{j} \wedge \cdots \wedge X_{p} \otimes a
\end{aligned}
$$

(Here, as usual, the notation $\hat{X}_{i}$ indicates that $X_{i}$ is omitted.) We also consider $\mathbb{C}$ as a trivial $\mathfrak{h}$-module, and denote the differential in the standard complex $C$. $(\mathfrak{h}, \mathbb{C})$ by $d^{\prime}$.

In order to prove that $H_{k}(\mathfrak{g}, A) \neq 0$, it suffices to find a $k$-cycle $z \in C_{k}(\mathfrak{g}, A)$ which is not a boundary. Note that $\bigwedge^{k} \mathfrak{g}=\bigwedge^{k} \mathfrak{h} \oplus E$, where

$$
E=\bigoplus_{i=1}^{k} \bigwedge^{i} \mathfrak{r} \otimes \bigwedge^{k-i} \mathfrak{h} .
$$

Fix $\eta \in \bigwedge^{k} \mathfrak{h}, \eta \neq 0$, and set $z=\eta \otimes 1 \in C_{k}(\mathfrak{g}, A)$. Since $\mathfrak{h}$ acts on $A$ by derivations, we have $X \cdot 1=0$ for each $X \in \mathfrak{h}$. Now it follows from (7) that $d(\eta \otimes 1)=\left(d^{\prime} \eta\right) \otimes 1$, i.e., only the second group of summands in (7) survives. On the other hand, since $\mathfrak{h}$ is semisimple, we have $H_{k}(\mathfrak{h}, \mathbb{C}) \neq 0$, i.e., the differential $d^{\prime}: \bigwedge^{k} \mathfrak{h} \rightarrow \bigwedge^{k-1} \mathfrak{h}$ is zero [7. Therefore $d(\eta \otimes 1)=\left(d^{\prime} \eta\right) \otimes 1=0$.

In order to prove that $\eta \otimes 1$ is not a boundary, note that $A$ has a canonical augmentation $\varepsilon_{A}: A \rightarrow \mathbb{C}$ defined by $\varepsilon_{A}=\varepsilon i_{1}$, where $\varepsilon$ is the counit of $\widehat{U}(\mathfrak{g})$, and $i_{1}: A \rightarrow \widehat{U}(\mathfrak{g})=A \widehat{\#} \widehat{U}(\mathfrak{h}), a \mapsto a \otimes 1$ is the canonical embedding. Clearly, the restriction of $\varepsilon_{A}$ to $U(\mathfrak{r})$ is precisely the counit of $U(\mathfrak{r})$. Now take $\xi \in\left(\bigwedge^{k} \mathfrak{g}\right)^{*}$ such that $\xi(\eta)=1$ and $\left.\xi\right|_{E}=0$. We then have $\left(\xi \otimes \varepsilon_{A}\right)(\eta \otimes 1)=1$. Let us show that $\xi \otimes \varepsilon_{A}$ vanishes on $\operatorname{Im} d$. To this end, consider the decomposition

$$
\bigwedge^{k+1} \mathfrak{g} \otimes A=\left(\mathfrak{r} \otimes \bigwedge^{k} \mathfrak{h} \otimes A\right) \oplus(F \otimes A),
$$

where

$$
F=\bigoplus_{i=2}^{k+1} \bigwedge^{i} \mathfrak{r} \otimes \bigwedge^{k+1-i} \mathfrak{h} .
$$

It follows from (7) that $d(F \otimes A) \subset E \otimes A$. By the same formula, for each $X \in \mathfrak{r}$ and each $a \in A$ we have $d(X \otimes \eta \otimes a)=\eta \otimes X a+w$ for some $w \in E \otimes A$. Since $\left.\xi\right|_{E}=0$, we see that $\xi \otimes \varepsilon_{A}$ vanishes on $E \otimes A$. On the other hand, we have $\varepsilon_{A}(X a)=\varepsilon_{U(\mathfrak{r})}(X) \varepsilon_{A}(a)=0$, and so $\left(\xi \otimes \varepsilon_{A}\right)(\eta \otimes X a)=0$. Together with (8), this implies that $\xi \otimes \varepsilon_{A}$ vanishes on $\operatorname{Im} d$, and so $\eta \otimes 1 \notin \operatorname{Im} d$. The rest is clear.

Combining Lemma 3.4, Lemma 3.5, and Proposition 1.4, we obtain the following.

Theorem 3.6. Let $\mathfrak{g}$ be a finite-dimensional Lie algebra such that $\widehat{U}(\mathfrak{g})$ is stably flat over $U(\mathfrak{g})$. Then $\mathfrak{g}$ is solvable. 


\section{ACKNOWLEDGMENTS}

The author is grateful to A. Ya. Helemskii and A. A. Dosiev for valuable discussions.

\section{REFERENCES}

[1] Bonneau, P., Flato, M., Gerstenhaber, M., Pinczon, G. The hidden group structure of quantum groups: strong duality, rigidity and preferred deformations, Comm. Math. Phys. 161 (1994), 125-156. MR1266072 (95b:17016)

[2] Doplicher, S., Kastler, D., Robinson, D. W. Covariance algebras in field theory and statistical mechanics. Comm. Math. Phys. 3 (1966), 1-28. MR0205095 (34:4930)

[3] Dosiev, A. A. Homological dimensions of the algebra formed by entire functions of elements of a nilpotent Lie algebra, Funct. Anal. Appl. 37 (1) (2003), 61-64. MR1988010(2004d:46054)

[4] Helemskii, A. Ya. The Homology of Banach and Topological Algebras, Moscow University Press, 1986 (Russian); English transl.: Kluwer Academic Publishers, Dordrecht, 1989. MR:1093462(92d:46178)

[5] Helemskii, A. Ya. Banach and Polynormed Algebras: General Theory, Representations, Homology, Nauka, Moscow, 1989 (Russian); English transl.: Oxford University Press, 1993. MR:1031991 (91h:46001)

[6] Kiehl, R. and Verdier, J. L. Ein einfacher Beweis des Kohärenzsatzes von Grauert, Math. Ann. 195 (1971), 24-50. MR0306555 (46:5678)

[7] Koszul, J.-L. Homologie et cohomologie des algèbres de Lie, Bull. Soc. Math. France $\mathbf{7 8}$ (1950), 65-127. MR0036511(12:120g)

[8] McConnell, J. C.; Robson, J. C. Noncommutative Noetherian Rings. John Wiley \& Sons, Ltd., Chichester, 1987. MR0934572 (89j:16023)

[9] Mitiagin, B.; Rolewicz, S.; Zelazko, W. Entire functions in $B_{0}$-algebras. Studia Math. 21 (1961/1962), 291-306. MR0144222 (26:1769)

[10] Neeman, A. and Ranicki, A. Noncommutative localization and chain complexes. I. Algebraic $K$ - and L-theory, Preprint arXiv.org:math.RA/0109118.

[11] Pirkovskii, A. Yu. Stably flat completions of universal enveloping algebras, Preprint arXiv.org:math.FA/0311492.

[12] Rieffel, M. A. Induced Banach representations of Banach algebras and locally compact groups. J. Functional Analysis 1 (1967), 443-491. MR0223496 (36:6544)

[13] Schweitzer, L. B. Dense m-convex Fréchet subalgebras of operator algebra crossed products by Lie groups. Internat. J. Math. 4 (4) (1993), 601-673. MR1232986 (94g:46058)

[14] Sweedler, M. E. Hopf algebras. Benjamin, New York, 1969. MR0252485 (40:5705)

[15] Taylor, J. L. Homology and cohomology for topological algebras, Adv. Math. 9 (1972), 137182. MR.0328624 (48:6966)

[16] Taylor, J. L. A general framework for a multi-operator functional calculus, Adv. Math. 9 (1972), 183-252. MR0328625(48:6967)

[17] Taylor, J. L. Functions of several noncommuting variables, Bull. Amer. Math. Soc. 79 (1973), 1-34. MR0315446 (47:3995)

Department of Nonlinear Analysis and Optimization, Faculty of Science, Peoples' Friendship University of Russia, Mikluho-Maklaya 6, 117198 Moscow, Russia

E-mail address: pirkosha@sci.pfu.edu.ru

E-mail address: pirkosha@online.ru 